\title{
Study of gas purifiers for the CMS RPC detector
}

\author{
L. Benussi ${ }^{\text {a }}$, S. Bianco ${ }^{\text {a }}$, S. Colafranceschi ${ }^{\text {a,c,b }}{ }^{\text {, F.L. Fabbri }}{ }^{\text {a }}$, F. Felli ${ }^{\text {a,b }}$, M. Ferrini ${ }^{\text {a,b }}$, M. Giardoni ${ }^{\text {a }}$, \\ T. Greci ${ }^{\text {a,b }}$, A. Paolozzi ${ }^{\text {a,b }}$, L. Passamonti ${ }^{a}$, D. Piccolo ${ }^{a}$, D. Pierluigi ${ }^{a}$, A. Russo $^{a}$, G. Saviano $^{\mathrm{a}, \mathrm{b}, *}$, \\ S. Buontempo ${ }^{d}$, A. Cimmino $^{\text {d,e }}$, M. de Gruttola ${ }^{\text {d,e }}$, F. Fabozzi $^{d}$, A.O.M. Iorio $^{\text {d,e }}$, L. Lista $^{d}$, P. Paolucci $^{\mathrm{d}}$, \\ P. Baesso ${ }^{\text {f }}$, G. Belli ${ }^{\text {, }}$ D. Pagano ${ }^{f}$, S.P. Ratti ${ }^{f}$, A. Vicini ${ }^{f}$, P. Vitulo ${ }^{f}$, C. Viviani ${ }^{f}$, R. Guida ${ }^{c}$, A. Sharma ${ }^{c}$ \\ a INFN Laboratori Nazionali di Frascati, Via E. Fermi 40, I-00044 Frascati, Italy \\ b Sapienza Università degli Studi di Roma "La Sapienza", Piazzale A. Moro, Roma, Italy \\ c CERN, CH-1211 Genéve 23, F-01631, Switzerland \\ d INFN Sezione di Napoli, Complesso Universitario di Monte Sant'Angelo, edificio 6, 80126 Napoli, Italy \\ e Università di Napoli Federico II, Complesso Universitario di Monte Sant'Angelo, edificio 6, 80126 Napoli, Italy \\ ${ }^{\mathrm{f}}$ INFN Sezione di Pavia and Università degli studi di Pavia, Via Bassi 6, 27100 Pavia, Italy
}

\section{A R T I C L E I N F O}

Available online 17 September 2010

Keywords:

RPC

CMS

Gas

Purifier detectors HEP muon

\begin{abstract}
A B S T R A C T
The CMS RPC muon detector utilizes a gas recirculation system called closed loop (CL) to cope with large gas mixture volumes and costs. A systematic study of CL gas purifiers has been carried out over 400 days between July 2008 and August 2009 at CERN in a low-radiation test area, with the use of RPC chambers with currents monitoring, and gas analysis sampling points. The study aimed to fully clarify the presence of pollutants, the chemistry of purifiers used in the $\mathrm{CL}$, and the regeneration procedure. Preliminary results on contaminants release and purifier characterization are reported.
\end{abstract}

(c) 2010 Elsevier B.V. All rights reserved.

\section{Introduction}

The Resistive Plate Chamber (RPC) [1] muon detector of the Compact Muon Solenoid (CMS) experiment [2] utilizes a gas recirculation system called closed loop (CL) $[3,4]$ to cope with large gas mixture volumes and costs [13-15]. A systematic study of closed loop gas purifiers has been carried out in 2008 and 2009 at the ISR experimental area of CERN with the use of RPC chambers exposed to cosmic rays with currents monitoring and gas analysis sampling points. Goals of the study [5] were to observe the release of contaminants in correlation with the dark current increase in RPC detectors, to measure the purifier lifetime, to observe the presence of pollutants and to study the regeneration procedure. Previous results had shown the presence of metallic contaminants, and an incomplete regeneration of purifiers [6,7].

The basic function of the CMS CL system is to mix and purify the gas components in the appropriate proportions and to distribute the mixture to the individual chambers. The gas mixture used is $95.2 \%$ of $\mathrm{C}_{2} \mathrm{H}_{2} \mathrm{~F}_{4}$ in its environmental-friendly version $\mathrm{R} 137 \mathrm{a}, 4.5 \%$ of $i \mathrm{C}_{4} \mathrm{H}_{10}$, and $0.3 \% \mathrm{SF}_{6}$ to suppress streamers and operate in saturated avalanche mode. Gas mixture is humidified at the $45 \% \mathrm{RH}$ (Relative Humidity) level typically to

\footnotetext{
* Corresponding author at: INFN Laboratori Nazionali di Frascati, Via E. Fermi 40, I-00044 Frascati, Italy. Tel.: + 390644585826 ; fax: +390644585618.

E-mail address: giovanna.saviano@uniroma1.it (G. Saviano).
}

balance ambient humidity, which affects the resistivity of highly hygroscopic bakelite, and to improve efficiency at lower operating voltage. The $\mathrm{CL}$ is operated with a fraction of fresh mixture continuously injected into the system. Baseline design fresh mixture fraction for CMS is $2 \%$, the test CL system was operated at $10 \%$ fresh mixture. The fresh mixture fraction is the fraction of the total gas content continuously replaced in the CL system with fresh mixture. The filter configuration is identical to the CMS experiment.

\section{Experimental setup and data sample}

In the CL system gas purity is guaranteed by a multistage purifier system:

- The purifier- 1 consisting of a cartridge filled with $5 \AA$ ( $10 \%$ ) and $3 \AA$ (90\%) Type LINDE [12] molecular sieve [11] based on Zeolite manufactured by ZEOCHEM [8].

- The purifier-2 consisting of a cartridge filled with $50 \% \mathrm{Cu}-\mathrm{Zn}$ filter type R12 manufactured by BASF [9] and 50\% Cu filter type R3-11G manufactured by BASF.

- The purifier-3 consisting of a cartridge filled with $\mathrm{Ni}^{\mathrm{AlO}_{3}}$ filter type 6525 manufactured by LEUNA [10].

The experimental setup (Fig. 1) is composed of a CL system and an open mode gas system. A detailed description of the CL, the 


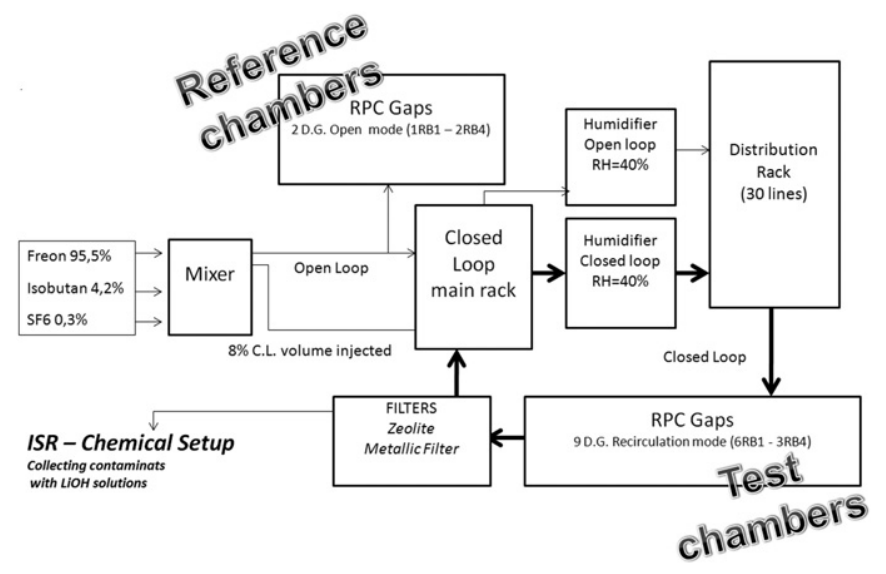

Fig. 1. Test setup with CL and open loop.

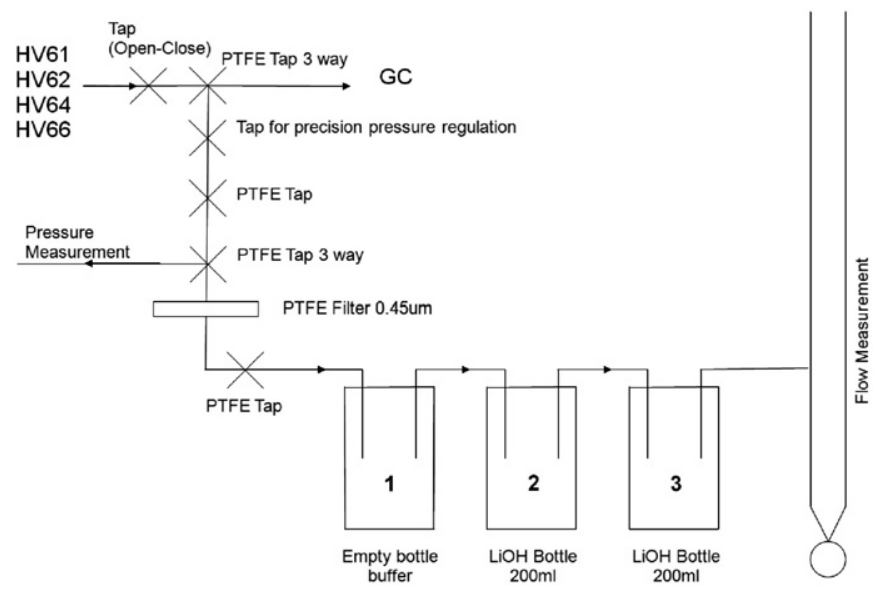

Fig. 2. Chemical setup.

experimental setup, and the filters studied can be found in Ref. [7]. The CL is composed of mixer, purifiers (in the subunit called "filters" in Fig. 1), recirculation pump and distribution to the RPC detectors. Eleven double-gap RPC detectors are installed, nine in CL and two in open mode. Each RPC detector has two gaps (upstream and downstream) whose gas lines are serially connected. The gas flows first in the upstream gap and then in the downstream gap. The detectors are operated at a $9.2 \mathrm{kV}$ power supply. The anode dark current drawn because of the high bakelite resistivity is approximately $1-2 \mu \mathrm{A}$. Gas sampling points before and after each filter in the closed loop allow gas sampling for chemical and gas chromatography analysis. The system is located in a temperature and humidity controlled hut, with online monitoring of environmental parameters.

Chemical analyses have been performed in order to study the dynamical behaviour of dark currents increase in the double-gap experimental setup and correlate to the presence of contaminants, measure lifetime of unused purifiers, and identify contaminant(s) in correlation with the increase of currents. In the chemical analysis setup (Fig. 2) the gas is sampled before and after each CL purifier, and bubbled into a set of PVC flasks. The first flask is empty and acts as a buffer, the second and third flasks contain $250 \mathrm{ml}$ solution of $\mathrm{LiOH}(0.001 \mathrm{~mol} / \mathrm{l}$ corresponding to $0.024 \mathrm{~g} / \mathrm{l}$, optimized to keep the $\mathrm{pH}$ of the solution at 11). The bubbling of gas mixture into the two flasks allows one to capture a wide range of elements that are likely to be released by the system, such as $\mathrm{Ca}, \mathrm{Na}, \mathrm{K}, \mathrm{Cu}, \mathrm{Zn}, \mathrm{Cu}, \mathrm{Ni}, \mathrm{F}$. At the end of each sampling line the flow is measured in order to have the total gas

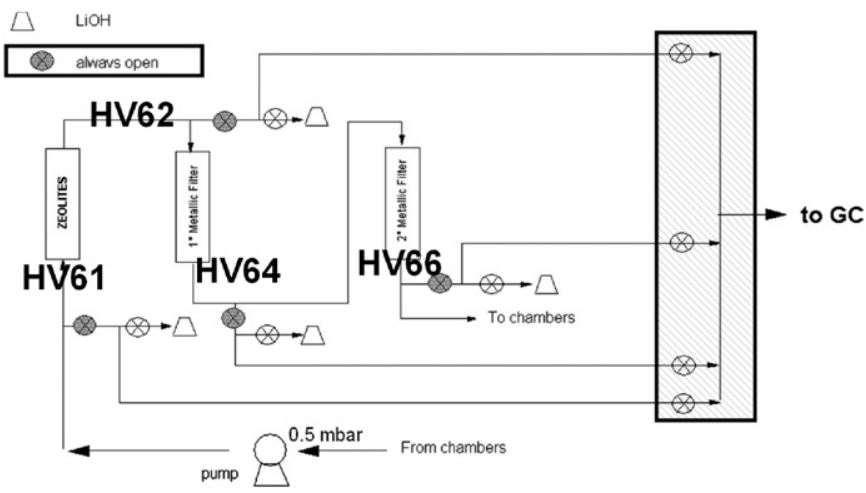

Fig. 3. Chemical setup sampling points.

amount for the whole period of sampling. Tygon filters $(0.45 \mu \mathrm{m})$ have been installed upstream the flasks.

The sampling points (Fig. 3) are located before the whole filters unit at position HV61, after purifier-1 (Zeolite) at HV62, after purifier-2 (Cu/Zn filter) at HV64 and after the Ni filter at position HV66. RPC are very sensitive to environmental parameters (atmospheric pressure, humidity, temperature), this study has been performed in environmentally controlled hut with pressure, temperature and relative humidity online monitoring. The comparison of temperature and humidity inside and outside the hut is displayed in Figs. 4 and 5, respectively, over the whole time range of the test. The inside temperature shows a variation of less than $\pm 0.5^{\circ} \mathrm{C}$; the inside humidity still reveals seasonal structures between $35 \%$ and $50 \%$, it is, however, much smaller than the variation outside.

Gas mixture composition was monitored twice a day by gas chromatography, which also provided the amount of air contamination, stable over the entire data taking run and below 300 (100) ppm in closed (open) loop. Purifiers were operated with unused filter material.

\section{Results and discussion}

The data-taking run was divided into cycles where different phenomena were expected. We have four cycles (Fig. 6), i.e., initial stable currents (cycles 1 and 2), at the onset of the raise of currents (cycle 3 ), in the full increase of currents (cycle 4). Cycle 4 was terminated in order not to damage permanently the RPC detectors. The currents of all RPC detectors in open loop were found stable over the four cycles. Fig. 6 shows the typical behaviour of one RPC detector in CL. While the current of the downstream gap is stable throughout the run, the current of the upstream gap starts increasing after about seven months. Such behaviour is suggestive of the formation of contaminants in the $\mathrm{CL}$ which are retained in the upstream gap, thus causing its current to increase, and leaving the downstream gap undisturbed. While the production of $\mathrm{F}^{-}$is constant during the run period, significant excess of $\mathrm{K}$ and $\mathrm{Ca}$ is found in the gas mixture in cycles 3 and 4 . The production of $\mathrm{F}^{-}$is efficiently depressed by the zeolite purifier (Fig. 7). The observed excess of $\mathrm{K}$ and $\mathrm{Ca}$ could be explained by a damaging effect of $\mathrm{HF}$ (continuously produced by the system) on the zeolite filter whose structure contains such elements. Further studies are in progress to confirm this model.

\section{Conclusions}

Preliminary results show that the lifetime of purifiers using unused material is approximately seven months. Contaminants 


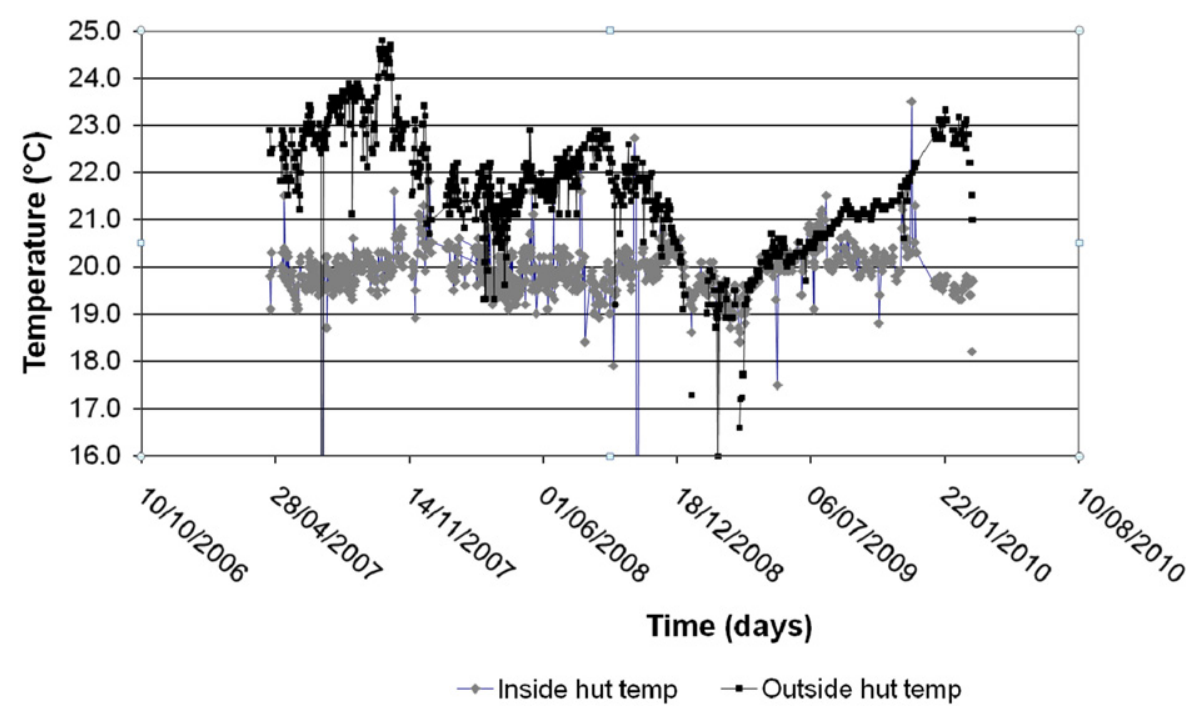

Fig. 4. Temperature distribution inside and outside the experimental hut.

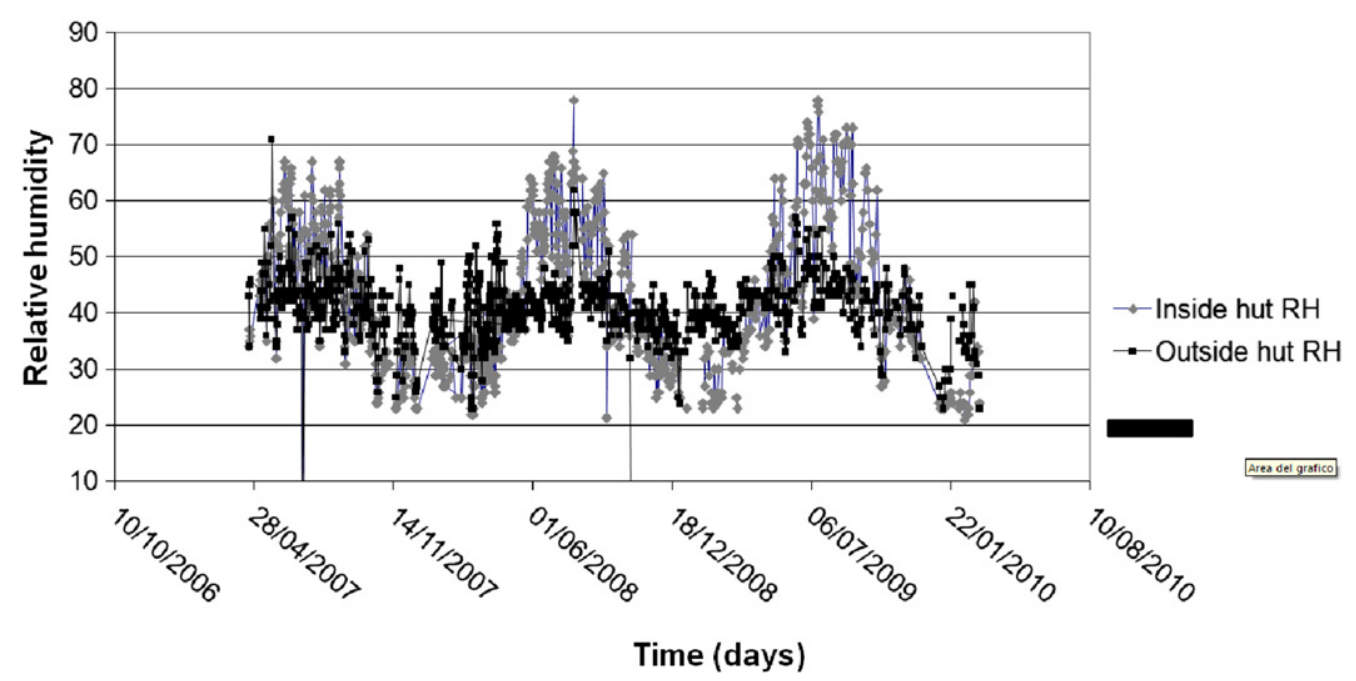

Fig. 5. Relative humidity inside and outside the experimental hut.
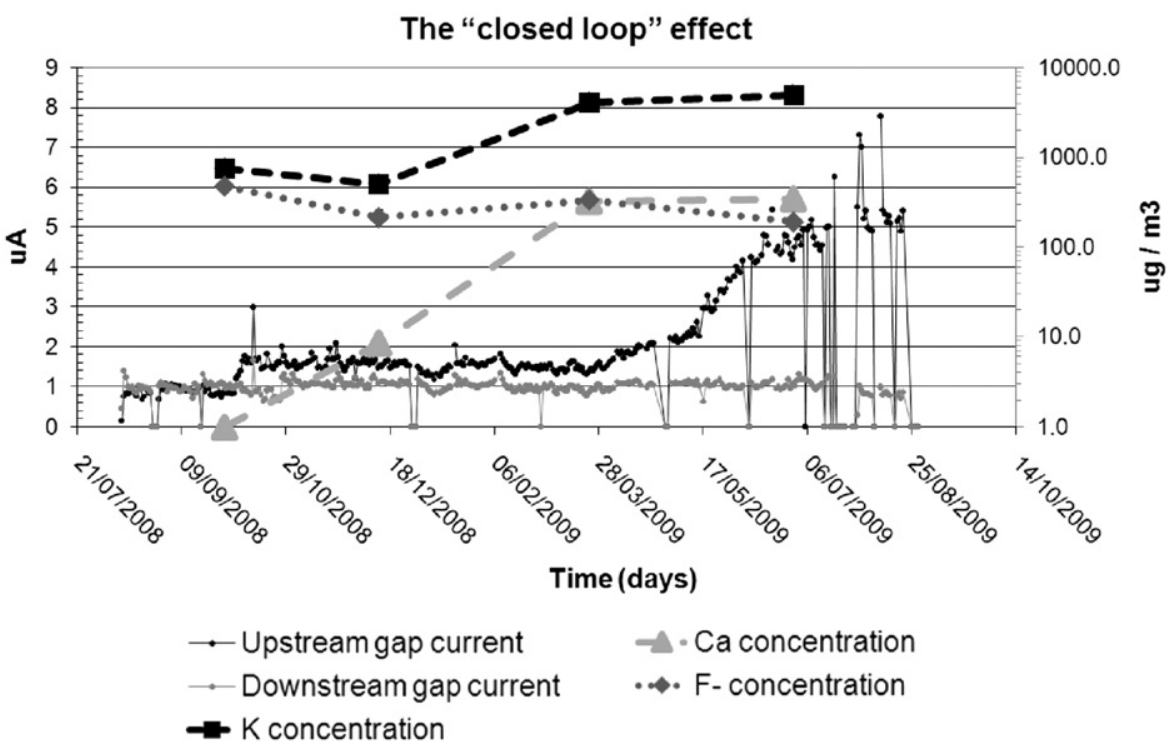

Fig. 6. Dark currents increase in the upstream gap and not in the downstream gap, correlated to the detection of contaminants in gas. 


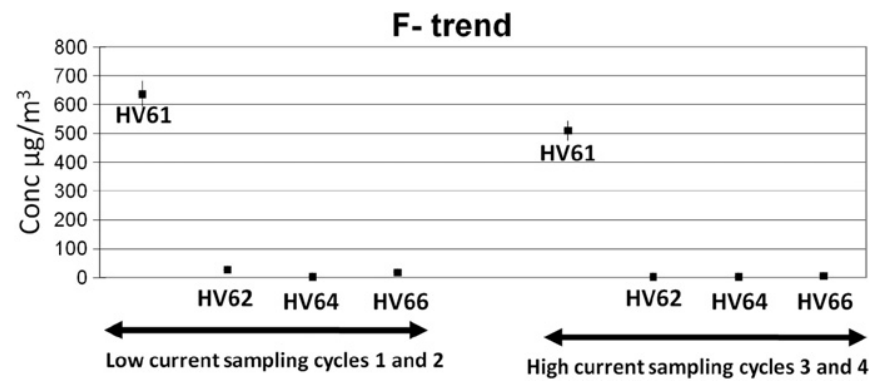

Fig. 7. Concentration of $\mathrm{F}^{-}$in gas as measured by the chemical setup. Sampling point HV61 is before the zeolite purifier, the others after each purifier.

$(\mathrm{K}, \mathrm{Ca})$ are released in the gas in correlation with the dark currents increase. The currents increase is observed only in the upstream gap. The study suggests that contaminants produced in the system stop in the upstream gap and affect its noise behaviour, leaving the downstream gap undisturbed. The presence of an excess production of $\mathrm{K}$ and $\mathrm{Ca}$ in coincidence with the currents increase suggests a damaging effect of HF produced in the system on the framework of zeolites which is based on $\mathrm{K}$ and $\mathrm{Ca}$. Further studies are in progress to fully characterize the system over the four cycles from the physical and the chemical point of view. The main goal is to better schedule the operation and maintenance of filters for the CMS experiment, where for a safe and reliable operation the filter regeneration is presently performed several times per week. A second run is being started with regenerated filter materials to measure their lifetime and confirm the observation of contaminants. Finally, studies in high-radiation environment at the CERN gamma irradiation facility are being planned.

\section{Acknowledgements}

The technical support of the CERN gas group is gratefully acknowledged. Thanks are due to F. Hahn for discussions, and to Nadeesha M. Wickramage, Yasser Assran for help in data taking shifts. This research was supported in part by the Italian Istituto Nazionale di Fisica Nucleare and Ministero dell' Istruzione, Università e Ricerca.

\section{References}

[1] R. Santonico, R. Cardarelli, Nucl. Instr. and Meth. 187 (1981) 377.

[2] CMS Collaboration, JINST 3 (2008) S08004

[3] M. Bosteels, et al., CMS gas system proposal, CMS Note 1999/018.

[4] L. Besset, et al., Experimental tests with a standard closed loop gas circulation system, CMS Note 2000/040.

[5] M. Abbrescia, et al., Proposal for a systematic study of the CERN closed loop gas system used by the RPC muon detectors in CMS, Frascati preprint LNF-06 27(IR), available at 〈http://www.lnf.infn.it/sis/preprint/ $\rangle$.

[6] G. Saviano, et al., Materials studies for the RPC detector in CMS, Presented at the RPC07 Conference, Mumbai, India, January 2008.

[7] S. Bianco, et al., Chemical analyses of materials used in the CMS RPC muon detector, CMS NOTE 2010/006.

[8] Manufactured by ZEOCHEM, 8708 Uetikon, Switzerland.

[9] BASF Technical Bulletin.

[10] LEUNA Data Sheet, September 9, 2003, Catalyst KL6526-T.

[11] GRACE Davison Molecular Sieves data sheet.

[12] LINDE Technical Bullettin.

[13] L. Benussi, et al., JINST 4 (2009) doi:10.1088 1748-0221 408 P08006 arXiv:0812.1710 [phys ics.ins-det].

[14] M. Abbrescia, et al., Nucl. Phys. Proc. Suppl. 158 (2006) 30 NUPHZ,158,30.

[15] G. Aielli, et al., Fluoride production in RPCs operated with F-compound gases, in: 8th Workshop on Resistive Plate Chambers and Related Detectors, Seoul, Korea, 10-12 October 2005. Nucl. Phys. Proc. Suppl. 158 (2006) 143. 